\title{
Crescimento de codornas de diferentes grupos genéticos por meio de modelos não lineares
}

\author{
[Growth comparison of different genetic groups using nonlinear models] \\ L.F.M. Mota ${ }^{1}$, D.C. Alcântara ${ }^{1}$, L.R.A. Abreu ${ }^{1}$, L.S. Costa ${ }^{1}$, A.V. Pires ${ }^{1}$, C.M. Bonafé $e^{1}$, \\ M.A. Silva ${ }^{2}$, S.R.F. Pinheiro ${ }^{1}$ \\ ${ }^{1}$ Universidade Federal dos Vales do Jequitinhonha e Mucuri - Diamantina, MG \\ ${ }^{2}$ Pesquisador Visitante Nacional Sênior - Capes \\ RESUMO
}

\begin{abstract}
Objetivou-se com o presente trabalho ajustar modelos não lineares para descrever o padrão de crescimento de genótipos de codornas de corte e de postura do nascimento aos 35 dias de idade. Foram utilizados dados de 1280 codornas machos e fêmeas, provenientes de sete genótipos de codornas de corte (EV1, EV2, UFV1, UFV2, UFV3, LF1, LF2) e um de postura. Todas as codornas foram pesadas a cada sete dias, do nascimento aos 35 dias de idade. Foram ajustados os modelos Brody, Gompertz, Logístico, Richards e von Bertalanffy aos dados de peso corporal de todos os genótipos. As análises estatísticas foram realizadas utilizando-se o PROC NLIN do SAS (Statistical Analysis System, versão 9.0). Os critérios empregados para a escolha do melhor modelo para descrever a curva de crescimento foram o coeficiente de determinação ajustado $\left(\mathrm{R}^{2}\right)$, o desvio padrão assintótico (DPA), o desvio médio absoluto dos resíduos (DMA), o índice assintótico (IA), o critério de informação bayesiano (BIC), o critério de Akaike (AIC) e o quadrado médio do erro (QME). Todos os modelos não lineares testados convergiram, com exceção do modelo Brody, que não convergiu para nenhum genótipo avaliado. O modelo Richards não convergiu para o genótipo postura. Os parâmetros da curva de crescimento estimados pelos modelos indicaram maior precocidade, em geral, do genótipo postura comparado aos demais genótipos. O modelo Richards apresentou superestimação do ponto de inflexão para todos os genótipos, exceto para o genótipo postura. Os modelos Gompertz, Logístico e von Bertalanffy são recomendados para descrever o crescimento de codornas de corte dos grupos genéticos em estudo. O modelo Brody não convergiu, por isso não é recomendado para descrever o crescimento dos grupos genéticos de codornas em estudo.
\end{abstract}

Palavras-chave: Coturnix coturnix, Coturnix japonica, curva de crescimento, peso à maturidade, parâmetro de crescimento

\section{ABSTRACT}

This work aimed to adjust nonlinear models to describe the growth pattern of meat type and posture quail genotypes from hatch to 35 days of age. A total of 1280 male and female quails from EV1, EV2, UFV1, UFV2, UFV3, LF1 and LF2 and Posture genotypes were weighed every seven days, from hatch to 35 days of age. Brody, Gompertz, Logistic, von Bertalanffy and Richards models were used to fit the growth of the genetic groups. Statistical analyses were performed using PROC NLIN of SAS (Statistical Analysis System, version 9.0). The criteria used to select the model showing the best goodness of fit to the growth of the genotypes were: Adjusted Coefficient of Determination $\left(R^{2}\right)$, Asymptotic Standard Deviation (DPA), Absolut Mean Deviation (DMA), Asymptotic Index (AI), Bayesian Information Criterion (BIC), Akaike Information Criterion (AIC) and Errors Mean Square (MSE). All nonlinear models converged for the studied genotypes, except Richard the model for the Posture genotype. In general, the estimated parameters for the growth curve using nonlinear models suggested higher precocity for the Posture genotype in comparison to meat type genotypes. The Richard model overestimates the inflection point for all genotypes, except for the Posture genotype. The Gompertz, Logistic and von Bertalanffy are recommended to describe the growth patterns of the studied quail genetic groups. The Brody model did not converge and is not recommended to describe the genetic growth of the studied groups.

Keywords: Coturnix coturnix, Coturnix japonica, growth curve, growth parameter, weight maturity

Recebido em 2 de março de 2014

Aceito em 27 de março de 2015

E-mail: flaviommota.zoo@gmail.com 


\section{INTRODUÇÃO}

O uso de modelos matemáticos para descrever funções biológicas do crescimento é bastante útil principalmente para seleção de animais, em que variáveis quantitativas são utilizadas para representar diversos fatores que influenciam o fenômeno. $\mathrm{O}$ crescimento corporal é determinado por fatores genéticos e não genéticos. A constituição genética do indivíduo inclui efeito aditivo e combinações genéticas não aditivas que determinam o crescimento. Essas combinações e suas interações com condições de ambiente, como clima, nutrição, fatores intrínsecos (sexo, idade, estado fisiológico, etc.) e fatores extrínsecos (efeitos maternos) determinam a expressão fenotípica final do crescimento (Arango e Van Vleck, 2002).

A velocidade de crescimento de um animal depende da espécie, e animais de pequeno porte apresentam taxas de crescimento maiores do que os animais de grande porte. As curvas de crescimento relacionam o peso do animal com sua idade, mediante o uso de modelos não lineares para condensar grande volume de informação em pequeno conjunto de parâmetros que podem ser interpretados biologicamente. A associação desses parâmetros às características produtivas e reprodutivas dos animais é considerada como grande ferramenta em programas de seleção. Os parâmetros apropriados das funções de crescimento podem ser usados também para predizer o crescimento e a maturidade dos animais (Tholon et al., 2012; Arango e Van Vleck, 2002).

O peso corporal dos animais avaliados em diferentes idades permite construir uma curva de crescimento, que apresenta geralmente uma forma sigmoide, a qual pode ser ajustada ou descrita por meio de linearização dos dados (Tholon et al., 2012). A utilização das funções Brody, Gompertz , Logística, Richard e von Bertalanffy possibilita o estudo do crescimento animal em idades adultas, e esses modelos não lineares permitem avaliar a correlação existente entre os pesos em diferentes idades e a obtenção de indivíduos adultos com menor tamanho corporal (parâmetro A), maior velocidade de ganho de peso (parâmetro $\mathrm{K}$ ), associados à diminuição da maturidade sexual e à menor porcentagem de gordura na carcaça (Tholon e Queiroz, 2007).

$\mathrm{O}$ estudo da curva de crescimento possibilita melhor avaliação da população, o que permite planejar mudanças no crescimento dos animais por meio de seleção e otimização das estratégias de alimentação, a fim de se priorizarem as necessidades nutricionais em cada fase de crescimento. Além disso, as linhagens respondem de formas diferentes aos processos de seleção e, assim, geram diferenças no perfil de crescimento dos animais, as quais acabam influenciando o tipo de curva que o animal irá apresentar do nascimento à maturidade.

Desta forma, objetivou-se com este trabalho comparar modelos não lineares para descrever o crescimento de codornas de corte e de postura do nascimento aos 35 dias de idade.

\section{MATERIAL E MÉTODOS}

O estudo foi realizado nas instalações do Setor de Avicultura da Universidade Federal dos Vales do Jequitinhonha e Mucuri (UFVJM), em Diamantina, MG. Foram utilizadas 1280 codornas de um dia, machos e fêmeas, provenientes de sete genótipos de codornas de corte (EV1, EV2, UFV1, UFV2, UFV3, LF1, LF2) e um de postura. Todas as codornas foram pesadas do nascimento aos 35 dias de idade, a cada sete dias.

Ao nascimento, as aves foram identificadas individualmente dentro do grupo genético, pesadas e aleatoriamente transferidas para os boxes. As codornas foram alojadas em oito boxes (repetições) de dimensões $2,00 \times 2,00 \mathrm{~m}$, sendo 20 aves de cada genótipo por boxe.

As aves foram criadas em piso com cama de maravalha, com aquecimento artificial por meio de lâmpadas infravermelho de $250 \mathrm{~W}$ até os 21 dias de idade. As codornas receberam água e dieta à vontade durante todo o período experimental. Foram utilizadas duas dietas, uma do nascimento aos 21 dias de idade e outra de 22 a 35 dias de idade, formuladas à base de milho e farelo de soja (Tab. 1). As dietas foram formuladas, conforme informações de composições e digestibilidade dos ingredientes apresentadas em Rostango et al. (2011). 
Tabela 1. Composição das dietas experimentais para genótipos de codornas do nascimento aos 35 dias de idade

\begin{tabular}{|c|c|c|}
\hline \multirow{2}{*}{ Ingrediente } & \multicolumn{2}{|c|}{ Composição alimentar } \\
\hline & 1 a 21 dias & 22 a 35 dias \\
\hline Milho grão & 50,6607 & 58,4863 \\
\hline Soja farelo $45 \% \mathrm{~PB}$ & 45,6154 & 37,7993 \\
\hline Calcário & 1,1208 & 0,9337 \\
\hline Fosfato bicálcico & 1,0422 & 0,8183 \\
\hline Sal comum & 0,3925 & 0,3441 \\
\hline DL-metionina (99\%) & 0,3595 & 0,1776 \\
\hline Óleo de soja & 0,3174 & 1,0407 \\
\hline Vitini-aves $^{1}$ & 0,200 & 0,200 \\
\hline Min-aves ${ }^{2}$ & 0,200 & 0,200 \\
\hline L-Lisina $\mathrm{HCl}(79 \%)$ & 0,0915 & 0,000 \\
\hline Total & 100,00 & 100,00 \\
\hline \multicolumn{3}{|c|}{ Composição calculada } \\
\hline PB (\%) & 25,0000 & 22,0000 \\
\hline $\mathrm{EM}(\mathrm{kcal} / \mathrm{kg})$ & 2,9000 & 3,0500 \\
\hline Cálcio (\%) & 0,8500 & 0,7000 \\
\hline Fósforo disp. (\%) & 0,3200 & 0,2700 \\
\hline Lisina digestível (\%) & 1,3700 & 1,0943 \\
\hline Met+Cis digestível (\%) & 1,0400 & 0,8000 \\
\hline Metinona digestível aves (\%) & 0,6886 & 0,4776 \\
\hline Arginina dig. aves & 1,6335 & 0,0000 \\
\hline Sódio (\%) & 0,1700 & 0,1500 \\
\hline Treonina digestível (\%) & 0,8529 & 0,7514 \\
\hline Triptofano digestível (\%) & 0,2950 & 0,2543 \\
\hline Valina digestível aves (\%) & 1,0709 & 0,0000 \\
\hline
\end{tabular}

${ }^{1}$ Suplemento vitamínico por kg de produto: vit. A 3.750.000UI; vit. D3, 750.000UI; vit. E 7500mg; vit K 3,1.000mg; vit.B1, 750mg; vit.B2 $1.500 \mathrm{mg}$; vit.B6, $1500 \mathrm{mg}$; vit.B12, $7.500 \mathrm{mcg}$; vit.C $12.500 \mathrm{mg}$; biotina $30 \mathrm{mg}$; niacina $10.000 \mathrm{mg}$; ácido fólico 375 ; ácido pantotênico $3.750 \mathrm{mg}$; colina $10.000 \mathrm{mg}$; metionina $400.000 \mathrm{mg}$. ${ }^{2}$ Suplemento mineral por kg de produto: selênio $45 \mathrm{mg}$; iodo $175 \mathrm{mg}$; ferro $12.525 \mathrm{mg}$; cobre $2.500 \mathrm{mg}$; manganês $19.500 \mathrm{mg}$; zinco $13.750 \mathrm{mg}$; prom. Prod $15.000 \mathrm{mg}$; coccidiostático $10.000 \mathrm{mg}$; antioxidante (BHT) $500 \mathrm{mg}$.

As análises estatísticas foram realizadas utilizando-se o PROC NLIN do SAS (Statistical Analysis System, versão 9.0.), pelo método dos quadrados mínimos ordinários com o algoritmo de Gauss-Newton. O critério de convergência para as estimativas foi considerado quando: $\left[\left(\mathrm{SQR}_{\mathrm{j}-1}-\mathrm{SQR}_{\mathrm{j}}\right) /\left(\mathrm{SQR}_{\mathrm{j}}+10^{-6}\right)\right] \leq 10^{-8}$, em que $\mathrm{SQR}_{\mathrm{j}}$ é a soma de quadrado residual na iteração $\mathrm{j}$.

Para estimar o crescimento dos animais em função da idade, foram ajustados os seguintes modelos não lineares às informações de pesoidade de cada genótipo (Sarmento et al., 2006):

Brody: $y_{t}=A\left(1-B e^{-k t}\right)+\varepsilon$;

Gompertz: $y_{t}=A e^{-B e^{-k t}}+\varepsilon$;

Logístico: $y_{t}=A\left(1-B e^{-K t}\right)^{-m}+\varepsilon$;

von Bertalanffy: $y_{t}=A\left(1-B e^{-K t}\right)^{3}+\varepsilon ; \quad \mathrm{e}$

Richards: $y_{t}=A\left(1-B e^{-K t}\right)^{m}+\varepsilon$. 
Nesses modelos, " $\mathrm{y}_{\mathrm{t}}$ " representa o peso corporal da codorna em determinada idade; " $t$ " expressa a idade em dias; "A" é o peso assintótico, ou seja, o peso corporal da ave adulta (quando $\mathrm{t} \rightarrow \infty$ ); "K" mede a variação na velocidade do crescimento e está associado à taxa de maturidade, indicando que animais com valores menores de " $\mathrm{K}$ " apresentam crescimento lento; "b" é a constante de integração, cuja função é modelar a forma da curva de crescimento, porém não tem interpretação biológica; " $m$ " é constante do modelo de Richards, sendo determinante na origem das outras funções não lineares.

Os critérios utilizados para a seleção dos modelos que mais se ajustaram ao crescimento dos genótipos foram: convergência do modelo; $\mathrm{R}_{\mathrm{aj}}^{2}$ - coeficiente de determinação ajustado; QME - quadrado médio do erro; AIC - valor do critério de Akaike; BIC - valor do critério de informação bayesiano; DMA - desvio médio absoluto; DPA - desvio padrão assintótico; e, IA - índice assintótico. O desvio padrão assintótico (DPA) foi calculado a partir da raiz quadrada do quadrado médio do resíduo de cada modelo. O desvio médio absoluto dos resíduos (DMA), conforme proposto por Sarmento et al. (2006), foi calculado como $D M A=\sum_{i=i}^{n}\left|y_{i}-\hat{y}\right| / n$.

O índice assintótico (IA), descrito por Ratkowsky (1990), combina os critérios DPA, DMA e $\mathrm{R}^{2}$, sendo os demais ponderados em relação a este, assim: $I A=(D P A+D M A)-R^{2}$. Quanto menor o índice, melhor o ajuste do modelo. Critério de Akaike (AIC) foi calculado pela expressão: $A I C=-2 \log$ like $-2 p$, em que: p é o número de parâmetros, e loglike é o valor do logaritmo da função de verossimilhança considerando-se as estimativas dos parâmetros. O critério de informação bayesiano (BIC) foi calculado por: $B I C=-2 \ln L+p \ln (n)$, em que: $L$ refere-se ao valor maximizado da função de verossimilhança do modelo estimado, $\mathrm{p}$ ao número de parâmetros e $\mathrm{n}$ ao número de observações utilizadas para ajustar a curva; ln é o logaritmo natural.

\section{RESULTADOS E DISCUSSÃO}

Os modelos Gompertz, Logístico, Richards e von Bertalanffy convergiram para os genótipos avaliados, exceto o modelo Richards para o genótipo de postura. Isto evidencia o fato de que, por não fixar o ponto de inflexão e por possuir um parâmetro adicional, este modelo apresenta maiores dificuldades no processo iterativo de ajustamento. O modelo Brody não convergiu para nenhum dos genótipos em estudo.

Com base nos critérios utilizados para avaliar a qualidade de ajuste (Tab. 2), os modelos apresentaram valores próximos, o que indica bom ajuste para descrever o crescimento dos grupos genéticos de codornas avaliados em função da idade.

Os menores valores do índice assintótico (IA) foram observados para o grupo genético EV1 entre os genótipos de corte e também para o genótipo postura. $\mathrm{O}$ maior valor foi observado no grupo UFV2. O menor valor do DMA foi observado no grupo genético EV1, e o maior valor para o grupo UFV2. Esse critério avalia a qualidade do ajuste: quanto menor, melhor é o ajuste. Para o parâmetro DPA, o menor valor foi observado para o grupo UFV2, e o maior para o grupo EV1 (Tab. 2).

Com base no DMA, foram observados pequenos desvios entre os modelos, com menores valores para o modelo Richards, resultado que indica melhor ajuste médio quando comparado aos modelos von Bertalanffy, Gompertz e Logístico. Drumond et al. (2013), ao compararem o crescimento de codornas de corte por modelos não lineares, observaram que menores valores para o desvio médio absoluto (DMA) indicariam o melhor modelo. Sarmento et al. (2006), ao avaliarem modelos não lineares para curva de crescimento de ovinos Santa Inês, também obtiveram menores valores de DMA para o modelo Richards, mas o modelo apresentou problemas quanto à convergência no processo iterativo.

Com base no coeficiente de determinação ajustado $\left(R_{\text {ajustado }}^{2}\right)$, todos os modelos se ajustaram de forma semelhante para todos os genótipos. Ao se analisar o QME constata-se pequena variação entre os modelos, o que sugere adequação desses para descrever o crescimento de tais genótipos. 
Tabela 2. Critérios de qualidade de ajuste para os modelos não lineares ajustados para descrever o crescimento de oito genótipos de codornas

\begin{tabular}{|c|c|c|c|c|c|c|c|c|}
\hline \multirow{2}{*}{ Modelo } & \multirow{2}{*}{ Genótipo } & \multicolumn{7}{|c|}{ Critério de qualidade de ajuste } \\
\hline & & $\mathrm{R}_{\mathrm{aj}}^{2}(\%)$ & QME & AIC & $\mathrm{BIC}$ & DMA & DPA & IA \\
\hline \multirow{8}{*}{ Gompertz } & EV1 & 97,46 & 207,7 & 8851,5 & 8863,5 & 9,03 & 74,02 & $-14,41$ \\
\hline & EV2 & 96,89 & 265,7 & 6304,9 & 6309,5 & 10,29 & 70,29 & $-16,30$ \\
\hline & UFV1 & 97,17 & 274,8 & 6042,3 & 6046,9 & 10,58 & 70,01 & $-16,58$ \\
\hline & UFV2 & 96,50 & 359,8 & 3686,2 & 3690,2 & 12,09 & 65,44 & $-18,97$ \\
\hline & UFV3 & 97,06 & 305,4 & 6126,3 & 6130,9 & 11,07 & 68,51 & $-17,48$ \\
\hline & LF1 & 96,45 & 294,3 & 6304,2 & 6308,8 & 10,93 & 68,36 & $-17,16$ \\
\hline & LF2 & 95,63 & 355,7 & 5380,6 & 5385,0 & 11,81 & 64,96 & $-18,86$ \\
\hline & Postura & 93,91 & 113,7 & 8547,6 & 8552,6 & 5,96 & 77,29 & $-10,66$ \\
\hline \multirow{8}{*}{ Logístico } & EV1 & 97,45 & 209,1 & 8858,2 & 8864,2 & 9,31 & 73,68 & $-14,46$ \\
\hline & EV2 & 96,87 & 266,8 & 6307,4 & 6312,1 & 10,51 & 70,02 & $-16,33$ \\
\hline & UFV1 & 97,15 & 276,2 & 6045,7 & 6050,3 & 10,86 & 69,67 & $-16,62$ \\
\hline & UFV2 & 96,49 & 360,9 & 3687,2 & 3691,3 & 12,41 & 65,08 & $-19,00$ \\
\hline & UFV3 & 97,04 & 306,8 & 6129,2 & 6133,8 & 11,34 & 68,18 & $-17,52$ \\
\hline & LF1 & 96,43 & 296,3 & 6308,7 & 6313,3 & 11,25 & 67,96 & $-17,21$ \\
\hline & LF2 & 95,60 & 357,1 & 5384,1 & 5388,5 & 12,05 & 64,65 & $-18,90$ \\
\hline & Postura & 93,90 & 113,8 & 8549,1 & 8554,2 & 6,09 & 77,14 & $-10,67$ \\
\hline \multirow{8}{*}{$\begin{array}{c}\text { von } \\
\text { Bertalanffy }\end{array}$} & EV1 & 97,43 & 209,8 & 8862,0 & 8867 & 9,26 & 73,68 & $-14,48$ \\
\hline & EV2 & 96,85 & 268,7 & 6312,6 & 6317,2 & 10,66 & 69,79 & $-16,39$ \\
\hline & UFV1 & 97,14 & 277,8 & 6312,6 & 6317,2 & 10,65 & 69,82 & $-16,67$ \\
\hline & UFV2 & 96,47 & 363,2 & 3689,9 & 3694,0 & 12,32 & 65,09 & $-19,06$ \\
\hline & UFV3 & 97,03 & 308,1 & 6132,4 & 6136,9 & 11,32 & 68,15 & $-17,55$ \\
\hline & LF1 & 96,44 & 295,2 & 6306,4 & 6311,0 & 11,32 & 67,93 & $-17,18$ \\
\hline & LF2 & 95,62 & 355,9 & 5380,9 & 5385,4 & 11,88 & 64,87 & $-18,87$ \\
\hline & Postura & 93,88 & 114,3 & 8553,4 & 8558,3 & 5,93 & 77,25 & $-10,69$ \\
\hline \multirow{8}{*}{ Richards } & EV1 & 97,46 & 207,8 & 8851,1 & 8856,1 & 8,98 & 74,06 & $-14,42$ \\
\hline & EV2 & 96,88 & 266,1 & 6304,8 & 6309,5 & 10,28 & 70,29 & $-16,31$ \\
\hline & UFV1 & 97,16 & 275,1 & 6042,2 & 6046,8 & 10,55 & 70,02 & $-16,59$ \\
\hline & UFV2 & 96,49 & 360,7 & 3686,2 & 3690,2 & 12,07 & 65,43 & $-18,99$ \\
\hline & UFV3 & 97,05 & 305,8 & 6126,2 & 6130,8 & 12,07 & 67,49 & $-17,49$ \\
\hline & LF1 & 96,45 & 294,3 & 6303,2 & 6307,8 & 10,84 & 68,45 & $-17,16$ \\
\hline & LF2 & 95,63 & 355,6 & 5379,5 & 5383,9 & 11,71 & 65,06 & $-18,86$ \\
\hline & Postura & - & - & - & - & - & - & - \\
\hline
\end{tabular}

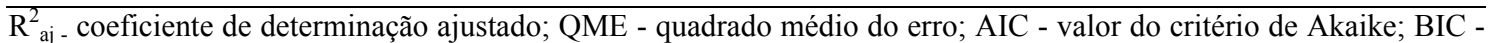
valor do critério de informação bayesiano; DMA - desvio médio absoluto; DPA - desvio padrão assintótico; IA índice assintótico.

Os modelos Logístico e von Bertalanffy, em geral, apresentaram maior variação do QME. Sarmento et al. (2006), ao avaliarem modelos de curva de crescimento em ovinos, observaram pequena variação no quadrado médio do resíduo para os modelos Brody e Logístico. Menores valores para os critérios BIC e AIC indicam melhor ajustamento em razão da redução do QME do modelo, o que sugere melhor ajuste aos dados para representar mudanças do peso em função do tempo.
O menor valor para o índice assintótico (IA) do modelo Gompertz indica seu melhor ajuste para os grupos genéticos de codornas. Este resultado corrobora os de Drumond et al. (2013), que avaliaram a curva de crescimento de codornas de corte e observaram que o modelo Gompertz apresentou menores valores de IA para machos. Araújo (2012) considera que o IA, por ser um critério que combina $\mathrm{R}^{2}$, DPA e DMA, é, em princípio, considerado um critério mais 
completo, sendo este critério recomendado para escolha de modelos.

Os baixos valores estimados para o índice assintótico (IA) foram próximos entre os modelos avaliados e indicam que eles apresentaram bom ajustamento para descrever o crescimento corporal de diferentes genótipos de codornas.

O modelo Richards (Tab. 3) superestimou o ponto de inflexão $(\mathrm{m})$ para os grupos genéticos em estudo. Isto mostra que tal modelo não se adequa à descrição dos pesos corporais dos genótipos estudados, o que pode ser explicado pela correlação alta e negativa entre os parâmetros b e m (Tab. 4). Sarmento et al. (2006) observaram que o modelo Richards, mesmo apresentando bom ajuste para representar a curva média, não deve ser escolhido, em razão de maiores dificuldades para atingir a convergência decorrentes de haver maior número de parâmetro a ser estimado e de esse modelo não fixar um ponto de inflexão.

Tabela 3. Estimativas dos parâmetros para os modelos de crescimentos de diferentes genótipos de codornas de corte e postura

\begin{tabular}{|c|c|c|c|c|c|}
\hline \multirow{2}{*}{ Modelo } & \multirow{2}{*}{ Genótipo } & \multicolumn{4}{|c|}{ Estimativa do parâmetro da curva } \\
\hline & & A & $\mathrm{b}$ & $\mathrm{k}$ & $\mathrm{M}$ \\
\hline \multirow{8}{*}{ Gompertz } & EV1 & 357,50 & 3,77 & 0,070 & - \\
\hline & EV2 & 361,40 & 3,8 & 0,070 & - \\
\hline & UFV1 & 381,60 & 3,83 & 0,070 & - \\
\hline & UFV2 & 410,50 & 3,88 & 0,068 & - \\
\hline & UFV3 & 409,68 & 3,81 & 0,068 & - \\
\hline & LF1 & 365,38 & 3,85 & 0,07 & - \\
\hline & LF2 & 361,41 & 3,85 & 0,07 & - \\
\hline & Postura & 166,39 & 3,17 & 0,072 & - \\
\hline \multirow{8}{*}{ Logístico } & EV1 & 331,93 & - & 0,085 & 5,11 \\
\hline & EV2 & 336,23 & - & 0,085 & 5,14 \\
\hline & UFV1 & 355,76 & - & 0,086 & 5,17 \\
\hline & UFV2 & 380,05 & - & 0,083 & 5,24 \\
\hline & UFV3 & 379,41 & - & 0,083 & 5,14 \\
\hline & LF1 & 336,57 & - & 0,083 & 5,22 \\
\hline & LF2 & 333,31 & - & 0,082 & 5,22 \\
\hline & Postura & 155,82 & - & 0,085 & 4,31 \\
\hline \multirow{8}{*}{ von Bertalanffy } & EV1 & 444,79 & 0,77 & 0,044 & - \\
\hline & EV2 & 446,52 & 0,77 & 0,044 & - \\
\hline & UFV1 & 469,13 & 0,77 & 0,045 & - \\
\hline & UFV2 & 518,67 & 0,78 & 0,043 & - \\
\hline & UFV3 & 514,31 & 0,78 & 0,043 & - \\
\hline & LF1 & 471,18 & 0,78 & 0,041 & - \\
\hline & LF2 & 462,76 & 0,78 & 0,041 & - \\
\hline & Postura & 194,53 & 0,69 & 0,047 & - \\
\hline \multirow{8}{*}{ Richards } & EV1 & 368,24 & 0,21 & 0,065 & 16,06 \\
\hline & EV2 & 364,47 & 0,07 & 0,069 & 51,82 \\
\hline & UFV1 & 389,07 & 0,16 & 0,068 & 22,74 \\
\hline & UFV2 & 413,97 & 0,07 & 0,067 & 57,49 \\
\hline & UFV3 & 419,07 & 0,16 & 0,065 & 21,48 \\
\hline & LF1 & 393,13 & 0,39 & 0,057 & 7,94 \\
\hline & LF2 & 397,94 & 0,49 & 0,055 & 6,16 \\
\hline & Postura & - & - & - & - \\
\hline
\end{tabular}

$\mathrm{A}=$ peso assintótico ou peso médio à maturidade; $\mathrm{b}=$ constante de integração sem interpretação biológica; $\mathrm{k}=$ taxa de crescimento; $\mathrm{e}, \mathrm{m}=$ ponto de inflexão. 
O modelo von Bertalanffy apresentou as maiores estimativas para o parâmetro " $\mathrm{A}$ " (peso à idade adulta) para os genótipos de codornas avaliados, em que os genótipos UFV2 e UFV3 apresentaram as maiores estimativas. $\mathrm{O}$ genótipo EV1 apresentou menor valor entre os genótipos de codornas de corte para o parâmetro "A". Drumond et al. (2013), ao avaliarem curvas de crescimento em codornas de corte, observaram que, entre os modelos estudados, o modelo von Bertalanffy estimou maiores pesos à idade adulta para o genótipo avaliado, obtendo-se, dessa forma, resultados semelhantes aos do presente estudo.

O parâmetro k (Tab. 3) representa a taxa de maturidade do animal e indica a velocidade de crescimento para se atingir o peso assintótico. Segundo Malhado et al. (2009), os animais com altos valores de $\mathrm{k}$ apresentam maior precocidade em relação aos animais com menores valores de " $k$ " e de peso inicial similar. Em decorrência da pequena variação no peso ao nascimento, a variação entre os valores de $\mathrm{k}$ representa, com boa precisão, as variações na velocidade relativa com que o animal cresce.

As menores estimativas do parâmetro " $\mathrm{k}$ " foram obtidas pelo modelo von Bertalanffy (Tab. 3), resultado da correlação negativa existente entre os parâmetros "A" e "k" (Tab. 4) também verificada por Garnero et al. (2005) e Drumond et al. (2013). Alguns autores observaram valores menores da taxa de maturidade $(\mathrm{k})$ para o modelo von Bertalanffy comparado aos modelos Gompertz, Logístico e Richards em outras espécies de animais (Garnero et al., 2005;
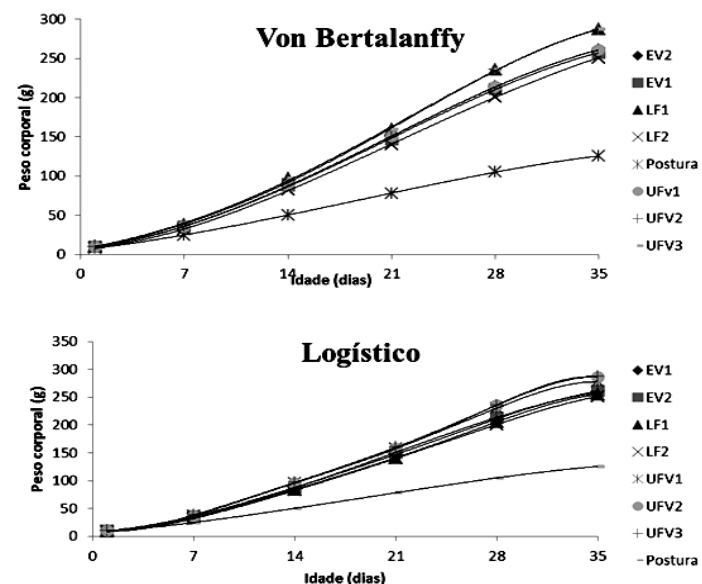

Sarmento et al., 2006; Souza et al., 2011; Tholon et al., 2012; Drumond et al., 2013).

Em geral, as correlações entre os parâmetros " $\mathrm{A}$ " e " $k$ " nas diferentes curvas são altas e negativas. A correlação negativa entre esses parâmetros indica que animais com maior velocidade de crescimento alcançam o peso à idade adulta mais cedo e apresentam menor probabilidade de atingirem pesos adultos mais altos que animais com taxas de maturidade menores e, é lógico, com crescimento mais lento e que apresentam maiores pesos à idade adulta. McManus et al. (2003) salientam que a relação biológica mais importante para uma curva está entre os parâmetros A e k. A correlação negativa existente entre esses parâmetros indica que animais com menores taxas de crescimento atingem maiores pesos à maturidade (Tab. 4), fato também observado por Drumond et al. (2013).

Observou-se que todos os genótipos apresentaram peso ao nascimento semelhante. A partir do sétimo dia de idade, os genótipos de corte começaram a mostrar diferenças de crescimento, entretanto o genótipo postura apresentou diferenças de crescimento antes do sétimo dia de idade (Fig. 1). Os genótipos UFV2, UFV3 e LF1 mostraram um padrão de curva similar do nascimento aos 35 dias de idade e apresentaram maior estimação do parâmetro "A" pelo modelo von Bertalanffy. O genótipo LF2 obteve a menor estimativa para peso à idade adulta entre os genótipos de corte. $\mathrm{O}$ genótipo postura apresentou as maiores diferenças de crescimento comparado aos genótipos de corte.
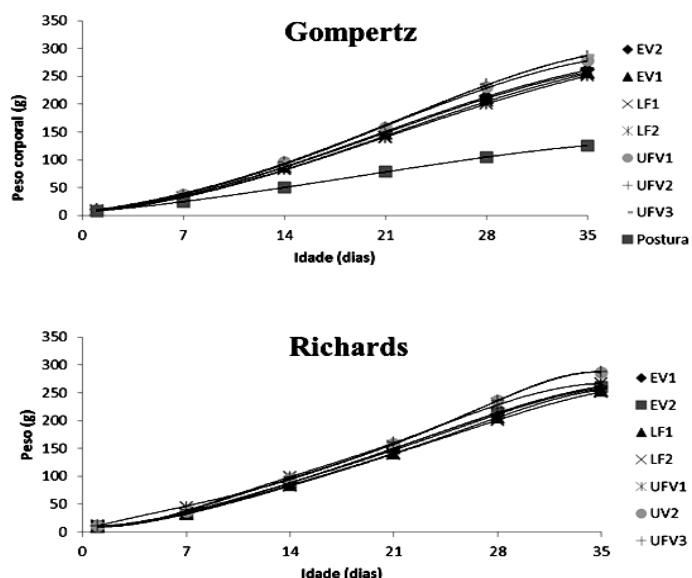

Figura 1. Curva de crescimento em função do peso corporal de codornas, segundo os modelos utilizados. 
Crescimento de codornas...

Tabela 4. Correlação fenotípica entre os parâmetros das curvas de crescimento

\begin{tabular}{|c|c|c|c|c|c|c|c|c|c|c|c|c|c|c|c|c|c|c|c|c|c|c|c|c|}
\hline \multicolumn{25}{|c|}{ Gompertz } \\
\hline & \multicolumn{3}{|c|}{ EV1 } & \multicolumn{3}{|c|}{ EV2 } & \multicolumn{3}{|c|}{ LF1 } & \multicolumn{3}{|c|}{ LF2 } & \multicolumn{3}{|c|}{ UFV1 } & \multicolumn{3}{|c|}{ UFV2 } & \multicolumn{3}{|c|}{ UFV3 } & \multicolumn{3}{|c|}{ Postura } \\
\hline & A & $\mathrm{b}$ & $\mathrm{k}$ & A & B & $\mathrm{k}$ & A & $\mathrm{b}$ & $\mathrm{k}$ & A & $\mathrm{b}$ & $\mathrm{K}$ & A & B & $\mathrm{k}$ & A & $\mathrm{b}$ & $\mathrm{K}$ & A & $\mathrm{b}$ & $\mathrm{k}$ & A & $\mathrm{b}$ & $\mathrm{k}$ \\
\hline A & 1 & & & 1 & & & 1 & & & 1 & & & 1 & & & 1 & & & 1 & & & 1 & & \\
\hline $\mathrm{b}$ & $-0,54$ & 1 & & $-0,56$ & 1 & & $-0,51$ & 1 & & $-0,53$ & 1 & & $-0,56$ & 1 & & $-0,56$ & 1 & & $-0,55$ & 1 & & $-0,42$ & 1 & \\
\hline $\mathrm{k}$ & $-0,94$ & 0,75 & 1 & $-0,94$ & 0,77 & 1 & $-0,95$ & 0,72 & 1 & $-0,95$ & 0,74 & 1 & $-0,94$ & 0,78 & 1 & $-0,95$ & 0,76 & 1 & $-0,95$ & 0,76 & 1 & $-0,94$ & 0,68 & 1 \\
\hline \multicolumn{25}{|c|}{ Logístico } \\
\hline & A & $\mathrm{k}$ & $\mathrm{m}$ & A & $\mathrm{K}$ & $\mathrm{m}$ & A & $\mathrm{k}$ & $\mathrm{m}$ & A & $\mathrm{k}$ & $\mathrm{M}$ & A & $\mathrm{K}$ & $\mathrm{m}$ & A & $\mathrm{k}$ & M & A & $\mathrm{k}$ & $\mathrm{m}$ & A & $\mathrm{k}$ & $\mathrm{m}$ \\
\hline A & 1 & & & 1 & & & 1 & & & 1 & & & 1 & & & 1 & & & 1 & & & 1 & & \\
\hline $\mathrm{k}$ & $-0,93$ & 1 & & $-0,93$ & 1 & & $-0,93$ & 1 & & $-0,93$ & 1 & & $-0,93$ & 1 & & $-0,93$ & 1 & & $-0,94$ & 1 & & $-0,92$ & 1 & \\
\hline $\mathrm{m}$ & $-0,47$ & 0,72 & 1 & $-0,49$ & 0,74 & 1 & $-0,45$ & 0,71 & 1 & $-0,48$ & 0,71 & 1 & $-0,5$ & 0,74 & 1 & $-0,5$ & 0,73 & 1 & $-0,49$ & 0,73 & 1 & $-0,36$ & 0,65 & 1 \\
\hline \multicolumn{25}{|c|}{ von Bertalanffy } \\
\hline & A & $\mathrm{b}$ & $\mathrm{k}$ & A & $\mathrm{B}$ & $\mathrm{k}$ & A & $\mathrm{b}$ & $\mathrm{k}$ & A & $\mathrm{b}$ & $\mathrm{K}$ & $\mathrm{A}$ & B & $\mathrm{k}$ & A & $\mathrm{b}$ & $\mathrm{K}$ & A & $\mathrm{b}$ & $\mathrm{k}$ & A & $\mathrm{b}$ & $\mathrm{k}$ \\
\hline A & 1 & & & 1 & & & 1 & & & 1 & & & 1 & & & 1 & & & 1 & & & 1 & & \\
\hline $\mathrm{b}$ & $-0,58$ & 1 & & $-0,61$ & 1 & & $-0,52$ & 1 & & $-0,57$ & 1 & & $-0,61$ & 1 & & $-0,61$ & 1 & & $-0,59$ & 1 & & $-0,43$ & 1 & \\
\hline $\mathrm{k}$ & $-0,98$ & 0,71 & 1 & $-0,97$ & 0,74 & 1 & $-0,98$ & 0,66 & 1 & $-0,98$ & 0,7 & 1 & $-0,97$ & 0,75 & 1 & $-0,98$ & 0,74 & 1 & $-0,98$ & 0,73 & 1 & $-0,97$ & 0,61 & 1 \\
\hline \multicolumn{25}{|c|}{ Richards } \\
\hline & A & $\mathrm{b}$ & $\mathrm{k}$ & A & $\mathrm{B}$ & $\mathrm{k}$ & A & $\mathrm{b}$ & k & A & $\mathrm{b}$ & $\mathrm{K}$ & A & B & $\mathrm{k}$ & A & $\mathrm{b}$ & $\mathrm{K}$ & A & $\mathrm{b}$ & $\mathrm{k}$ & A & $\mathrm{b}$ & $\mathrm{k}$ \\
\hline A & 1 & & & 1 & & & 1 & & & 1 & & & 1 & & & 1 & & & 1 & & & 1 & & \\
\hline $\mathrm{b}$ & 0,90 & 1 & & 0,91 & 1 & & 0,91 & 1 & & 0,91 & 1 & & 0,91 & 1 & & 0,91 & 1 & & 0,91 & 1 & & - & 1 & \\
\hline $\mathrm{k}$ & $-0,97$ & $-0,97$ & 1 & $-0,98$ & $-0,97$ & 1 & $-0,98$ & $-0,97$ & 1 & $-0,98$ & $-0,97$ & 1 & $-0,97$ & $-0,96$ & 1 & $-0,97$ & $-0,97$ & 1 & $-0,97$ & $-0,97$ & 1 & - & - & 1 \\
\hline $\mathrm{m}$ & $-0,91$ & $-0,99$ & 0,97 & $-0,91$ & $-0,99$ & 0,97 & $-0,92$ & $-0,99$ & 0,97 & $-0,92$ & $-0,99$ & 0,97 & $-0,91$ & $-0,99$ & 0,97 & $-0,91$ & $-0,99$ & 0,97 & $-0,91$ & $-0,99$ & 0,97 & - & - & - \\
\hline
\end{tabular}

biológica; " $k$ " = taxa de crescimento; e " $m$ "= ponto de inflexão.

Maiores estimativas do parâmetro "A", segundo o modelo Gompertz, foram obtidas para os grupos genéticos UFV2 e UFV3. Esse resultado indica que tais genótipos apresentam maiores pesos à idade adulta. O genótipo UFV1 apresentou diferença de crescimento em relação aos outros grupos UFV a partir de 21 dias de idade. Por outro lado, os genótipos EV1, EV2, LF1 e LF2 obtiveram padrões de crescimento parecidos e menores valores do peso à maturidade comparados aos genótipos UFV e apresentaram o menor peso à idade adulta, resultado decorrente de diferentes números de gerações de seleção para peso. Quanto ao parâmetro "k", o maior valor foi observado para o genótipo UFV1, e o menor valor observado para o EV1. Como " $k$ " indica a velocidade com que o animal passa pelo período de crescimento até se tornar adulto, conclui-se que o grupo UFV1 possui maior velocidade de crescimento em relação aos demais genótipos estudados.

Da mesma forma como observado no modelo Gompertz, segundo o modelo Logístico, os genótipos UFV2 e UFV3 apresentaram maiores valores do parâmetro "A" (peso corporal assintótico) enquanto os genótipos EV1, EV2, LF1 e LF2 obtiveram estimativas de peso corporal à idade adulta próximas, com menores estimativas do parâmetro "A", entre os genótipos de corte avaliados. O genótipo postura mostrou comportamento de crescimento diferente daqueles dos genótipos de corte em razão de as codornas de postura apresentarem menor peso corporal pelo fato de a seleção para este genótipo ser direcionada para maior produção de ovos e menor peso corporal.

A correlação entre ' $m$ ' e ' $K$ ' variou de média a alta e indica que quanto maior a inclinação da curvatura das funções, maior será a precocidade dos animais (Tholon e Queiroz, 2009). As correlações entre o peso médio à maturidade $(\mathrm{A})$ e o ponto de inflexão (m) foram negativas, variando de média a alta magnitude. Esse parâmetro " $\mathrm{m}$ " dá forma à curva e, consequentemente, determina em que proporção do peso à maturidade $(\mathrm{A})$ ocorre o ponto de inflexão da curva.

Considerando-se o modelo de Richards, os genótipos UFV3 e UFV2 apresentaram as maiores estimativas para peso aos 35 dias; já o genótipo UFV1 apresentou a menor estimativa para peso aos 35 dias em relação aos grupos genéticos do UFV, e as menores estimativas para peso aos 35 dias foram dos genótipos de UFV1, EV1, EV2, LF1 e LF2. 
Os genótipos que apresentaram menores estimativas de peso à idade adulta mostraram padrão de crescimento parecido, exceto o genótipo UFV1, que obteve um padrão de crescimento diferente. Entre o $28^{\circ}$ e o $35^{\circ}$ dia de idade as aves desse genótipo começaram a apresentar taxa de crescimento mais próxima aos genótipos EV1, EV2, LF1 e LF2. O maior valor do parâmetro " $\mathrm{k}$ " foi observado no grupo genético EV2, e o menor valor no grupo LF2, indicando que o primeiro genótipo apresenta taxa de crescimento mais rápida em relação aos genótipos avaliados (Tab. 4).

\section{CONCLUSÕES}

O modelo Broody não apresentou convergência. Os modelos não lineares Gompertz, Logístico e von Bertalanffy permitiram predizer a taxa de crescimento e o peso à idade adulta de todos os diferentes genótipos de codornas avaliados. O modelo Richards não deve ser indicado para representar o crescimento de codornas, pois, de modo geral, superestima o ponto de inflexão e não apresenta convergência para o grupo de postura.

\section{AGRADECIMENTOS}

Os autores agradecem o apoio financeiro recebido do $\mathrm{CNPq}$, da Capes e da Fapemig.

\section{REFERÊNCIAS}

ARAÚJO, R.O.; MARCONDES, C.R.; DAMÉ, M.C.F. et al. Classical nonlinear models to describe the growth curve for Murrah buffalo breed. Cienc. Rural, v.42, p.520-525, 2012.

ARANGO, J.A.; VAN VLECK, L.D. Size of beef cows: early ideas, new developments. Gen. Mol. Res., v.50, p.51-63, 2002.

DRUMOND, E.S.C.; GONÇALVES, F.M.; VELOSO, R.C. et al. Curvas de crescimento para codornas de corte. Ciênc. Rural, v.43, p. 1872-1877, 2013.

GARNERO, A.V.; MARCONDES, C.R.; BEZERRA, L.A.F. et al. Parâmetros genéticos da taxa de maturação e do peso assintótico de fêmeas da raça Nelore. Arq. Bras. Med. Vet. Zootec., v.57, p.652-662, 2005.
MALHADO, C.H.M.; CARNEIRO, P.L.S.; MELLO, P.R.A.M. et al. Growth curves in Dorper sheep crossed with the local Brazilian breeds, Morada Nova, Rabo Largo, and Santa Inês. Small Ruminant Res., v.84, p.16-21, 2009.

McMANUS, C.; EVANGELISTA, C.; FERNANDES, L.A.C. et al. Curvas de crescimento de ovinos bergamácia criados no Distrito Federal. Rev. Bras. Zootec., v.32, p.1207-1212, 2003.

RATKOWSKY, D.A. Handbook of nonlinear regression models. New York: Marcel Dekker, 1990. 241p.

ROSTAGNO, H.S.; ALBINO, L.F.T.; DONZELE J.L. et al. Tabelas brasileiras para aves e suínos: composição de alimentos e exigências nutricionais. 3.ed. Viçosa: Universidade Federal de Viçosa, 2011. 252p.

SARMENTO, J.L.R.; REGAZZI, A.J.; SOUSA, W.H. et al. Estudo da curva de crescimento de ovinos Santa Inês. Rev. Bras. Zootec., v.35, p.435-442, 2006.

SOUZA, L.A.; CARNEIRO, P.L.S.; MALHADO, C.H.M. et al. Curvas de crescimento em ovinos da raça morada nova criados no estado da Bahia. Rev. Bras. Zootec., v.40, p.1700-1705, 2011.

STATISTICAL analysis system, versão 9.0. Cary, NC: SAS, 2002.

THOLON, P.; QUEIROZ, S.A. Models for the analysis of growth curves for rearing tinamous (Rhynchotus rufescens) in captivity. Braz. J. Poult. Sci., v.9, p.23-31, 2007.

THOLON, P.; QUEIROZ, S.A. Modelos matemáticos utilizados para descrever curvas de crescimento em aves aplicados ao melhoramento genético animal. Ciênc. Rural, v.39, p.22612269, 2009.

THOLON, P.; PAIVA, R.D.M.; MENDES, A.R.A.; BARROZO, D. Utilização de funções lineares e não lineares para ajuste do crescimento de bovinos Santa Gertrudis, criados a pasto. ARS Vet., v.28, p.234-239, 2012. 